\title{
Avances metodológicos en el análisis de la concentración diaria de la precipitación en la España peninsular
}

\author{
Aziz Benhamrouche y Javier Martín Vide \\ Grupo de Climatología, Universidad de Barcelona \\ jmartinvide@ub.edu
}

Recibido: 4 de Octubre de 2011

Enviado a evaluar: 10 de Febrero de 2012

Aceptado: 23 de Marzo de 2012

\section{RESUMEN}

En el presente trabajo, partiendo del índice de concentración diaria de la precipitación de Martin-Vide (2004), basado en clases de $1 \mathrm{~mm}$ de longitud, se analiza la concentración de la precipitación en 32 observatorios de la España peninsular con índices similares usando clases de 5 y de $10 \mathrm{~mm}$, así como con los índices de Gini construidos con las mismas longitudes de clase. Se comparan los mapas resultantes para el período 1951-2010 y los subperíodos 1951-1980 y 1981-2010. Finalmente, con los índices citados se analizan las variaciones en la concentración diaria de la precipitación al pasar del primer al segundo subperíodo.

Palabras clave: Curva exponencial, índice de concentración, índice de Gini, España, precipitación diaria.

\section{Methodological advances in the analysis of the daily concentration of rainfall in mainland Spain}

\begin{abstract}
The daily concentration of the precipitation in 32 meteorological stations in peninsular Spain by means of the Concentration Index (Martin-Vide, 2004) and similar indexs using 5 and $10 \mathrm{~mm}$ classes, and the corresponding Gini's indexs, has been analysed. The maps for the period 1951-2010, as well as for the subperiods 1951-1980 and 1981-2010, have been compared. Finally, the changes in the daily concentration of the precipitation between the first and the second subperiods are studied.
\end{abstract}

Key words: Concentration Index, daily precipitation, exponential curve, Gini’s Index, Spain.

\section{Progrès méthodologiques dans l'analyse de la concentration journalière desprécipitations en Espagne peninsulaire}

\section{RÉSUMÉ}

Le présent travail a pour objectif l'analyse à partir de l'indice de concentration quotidienne des précipitations de Martin-Vide (2004), basé sur des classes de $1 \mathrm{~mm}$ de longueur, la concentration des précipitations de 32 observatoires d'Espagne péninsulaire avec des indices similaires de 5 et $10 \mathrm{~mm}$, ainsi que les indices de Gini construits avec les mêmes longueurs. Nous comparons les cartes qui en résultent pour la 
période 1951-2010 et sous-périodes de 1951-1980 et de 1981-2010. Enfin, avec les indices indiqués, on analyse les variations de la concentration quotidienne des précipitations allant du premier à la deuxième sous-période.

Mots-clés: Courbe exponentiel, indice de concentration, indice de Gini, Espagne, précipitation quotidienne.

\section{INTRODUCCIÓN}

Es bien conocida la alta concentración temporal de la precipitación en los climas mediterráneos, en los que en pocos días, e incluso en unas horas, se recoge un elevado porcentaje del total anual. Esto da lugar a que el carácter pluviométrico, lluvioso o seco, de un año dependa de unos pocos días particularmente lluviosos. No es raro que tras varios meses con escasas precipitaciones, conformando una secuencia de indigencia pluviométrica o una auténtica sequía (Gil Olcina y Olcina, 1997; Pita, 2001; Olcina, 2006), el carácter pluviométrico del año en curso cambie radicalmente con dos o tres episodios de precipitaciones torrenciales (Martín-Vide y Olcina, 2001). De esta manera, el año podría calificarse de seco, por este carácter predominante durante varios o muchos meses, y, al tiempo, de lluvioso, si sólo se atiende al total anual, que, gracias a los referidos episodios torrenciales, pudo sobrepasar con holgura el promedio anual.

La precipitación en el mundo mediterráneo muestra un carácter compulsivo, nula durante muchos días y con elevada intensidad durante unas horas o minutos. Su carácter convectivo y torrencial, especialmente en la mitad cálida del año y hasta bien avanzado el otoño, explica la brevedad y, al tiempo, la frecuente elevada intensidad de los chubascos. Los efectos de la alta concentración temporal de la precipitación se concretan, entre otros, en una alta erosividad del fenómeno sobre el suelo, más si se tienen en cuenta las débiles cubiertas vegetales de muchas comarcas; abundantes escorrentías súbitas, cuando no anegamientos e inundaciones rápidas (flash floods); una difícil gestión de los recursos hídricos, por la irregular entrada de agua en los sistemas hidráulicos y la imposibilidad de determinar el final de las sequías; etc.

Cuando se habla del Mediterráneo y del clima mediterráneo hay que hacer un matiz importante para el caso de la Península Ibérica. La alta concentración de la precipitación y las mayores intensidades diarias y horarias de la misma no se dan en el sur, que presenta el carácter subtropical (o genéricamente mediterráneo) más nítido, sino en la franja oriental, por la influencia de las aguas de la cuenca del mar Mediterráneo. Es la componente del mar Mediterráneo, más que la subtropicalidad (o mediterraneidad en sentido genérico) el factor decisivo en los extremos de intensidad y concentración pluviométrica (Martín-Vide, 2011). Y así lo han mostrado los mapas de intensidad de la precipitación (Elías Castillo y Ruiz Bertrán, 1979, Beguería et al., 2009) o los análisis de concentración diaria (Martín-Vide, 1987; Martin-Vide, 2004; Sánchez-Lorenzo y Martín-Vide, 2006).

En el presente trabajo, partiendo del índice de concentración (Concentration Index) de (Martin-Vide, 2004), se propone, en primer lugar, algunas modificaciones del 
mismo con vistas a un cálculo más rápido de la concentración pluviométrica diaria y para poder usar un mayor número de observatorios, dado que el citado índice es muy sensible a la calidad de los datos de partida, al exigir la contabilización de todas las cantidades diarias de lluvia, por pequeñas que sean, y su encuadre en clases muy finas, de $1 \mathrm{~mm}$ de longitud. Tras las nuevas propuestas del índice, se comparan los mapas resultantes para el período 1951-2010 y los subperíodos 1951-1980 y 19812010 , y se evalúa su interés y uso. En segundo lugar, se analizan los cambios habidos en la concentración diaria al pasar del primer al segundo subperíodo.

\section{METODOLOGÍA:LACONCENTRACIÓNDIARIADELAPRECIPITACIÓN, EL ÍNDICE DE CONCENTRACIÓN (CI) Y OTROS ALTERNATIVOS. HIPÓTESIS}

Es sabido que la distribución de frecuencias de las cantidades diarias de precipitación es ajustable mediante curvas exponenciales negativas (Brooks y Carruthers, 1953), dado que existen muchos días con precipitaciones escasas, sean inferiores a $1 \mathrm{~mm}$ y pocos con precipitaciones altas, descendiendo la frecuencia de forma exponencial. Con el objeto de evaluar la contribución relativa de los días más lluviosos el tercer coautor propuso un índice de concentración diaria de la precipitación (Concentration Index, CI) (Martin-Vide, 2004), similar al índice de Gini aplicado a las llamadas en Estadística curvas de Lorenz. Para ello se clasifican las cantidades diarias de precipitación en clases de longitud $1 \mathrm{~mm}$, comenzando por la [0,1-0,9], en orden creciente, [1,0-1,9], [2,0-2,9], etc. La distribución de frecuencias resultante muestra claramente una forma exponencial negativa. A continuación se procede de la forma que resume el cuadro 1, donde se presenta el ejemplo de Alicante (período 1951-2010). En la primera columna se sitúan las clases mencionadas, hasta la que contiene la cantidad diaria de precipitación más alta registrada, y en la segunda, las marcas de las clases. En la tercera columna, $n_{i}$, se expresan las frecuencias absolutas de cada clase y en la cuarta, $\Sigma n_{i}$, las frecuencias absolutas acumuladas, siendo el último valor el número total de días de precipitación de todo el período de estudio. Los valores de la quinta columna, $P_{i}$, se obtienen multiplicando, clase a clase, los de la segunda columna por los de la tercera, siendo así la cantidad total de precipitación aportada por cada clase (aproximadamente, dado que se ha sustituido cada valor por la marca de la clase). La sexta columna, $\Sigma P_{i}$, contiene los valores acumulados de la anterior columna, siendo el valor de la última clase la cantidad total de precipitación registrada durante el período de estudio. Por último, en las séptima y octava columnas se indican los porcentajes de los valores de las columnas cuarta y sexta, respecto a los valores de sus últimas filas, respectivamente, $\Sigma n_{i}(\%)=X$ y $\Sigma P_{i}(\%)=Y$. La representación de los valores de las últimas columnas, $(X, Y)$, da lugar a una línea poligonal marcadamente exponencial positiva, llamada curva de concentración, o de Lorenz. Sobre ella puede calcularse el índice de Gini, IG. En la figura 1 se presentan dos curvas de concentración, o de Lorenz, con índices de Gini apreciablemente diferentes. 
Cuadro 1: Ejemplo de los cálculos previos para la obtención del índice de concentración, CI (y del índice de Gini, IG). Caso de Alicante para el período 1951-2010

\begin{tabular}{|c|c|c|c|c|c|c|c|}
\hline lim.sup. & ma & ni & $\Sigma \mathrm{ni}$ & $\mathbf{P i}$ & $\Sigma \mathbf{P i}$ & $\begin{array}{c}\Sigma \text { ni }(\%) \\
=X\end{array}$ & $\begin{array}{c}\boldsymbol{\Sigma} \mathbf{P i}(\%) \\
=Y\end{array}$ \\
\hline 0,9 & 0,5 & 1380 & 1380 & 690 & 690 & 37,80 & 3,38 \\
\hline 1,9 & 1,5 & 547 & 1927 & 820,5 & 1510,5 & 52,78 & 7,41 \\
\hline 2,9 & 2,5 & 318 & 2245 & 795 & 2305,5 & 61,49 & 11,31 \\
\hline 3,9 & 3,5 & 239 & 2484 & 836,5 & 3142 & 68,04 & 15,41 \\
\hline 4,9 & 4,5 & 151 & 2635 & 679,5 & 3821,5 & 72,17 & 18,74 \\
\hline 5,9 & 5,5 & 136 & 2771 & 748 & 4569,5 & 75,90 & 22,41 \\
\hline 6,9 & 6,5 & 101 & 2872 & 656,5 & 5226 & 78,66 & 25,63 \\
\hline 7,9 & 7,5 & 85 & 2957 & 637,5 & 5863,5 & 80,99 & 28,75 \\
\hline 8,9 & 8,5 & 92 & 3049 & 782 & 6645,5 & 83,51 & 32,59 \\
\hline 9,9 & 9,5 & 63 & 3112 & 598,5 & 7244 & 85,24 & 35,52 \\
\hline 10,9 & 10,5 & 50 & 3162 & 525 & 7769 & 86,61 & 38,10 \\
\hline 11,9 & 11,5 & 41 & 3203 & 471,5 & 8240,5 & 87,73 & 40,41 \\
\hline 12,9 & 12,5 & 43 & 3246 & 537,5 & 8778 & 88,91 & 43,05 \\
\hline 13,9 & 13,5 & 32 & 3278 & 432 & 9210 & 89,78 & 45,17 \\
\hline 14,9 & 14,5 & 25 & 3303 & 362,5 & 9572,5 & 90,47 & 46,94 \\
\hline 15,9 & 15,5 & 27 & 3330 & 418,5 & 9991 & 91,21 & 49,00 \\
\hline 16,9 & 16,5 & 26 & 3356 & 429 & 10420 & 91,92 & 51,10 \\
\hline 17,9 & 17,5 & 22 & 3378 & 385 & 10805 & 92,52 & 52,99 \\
\hline 18,9 & 18,5 & 23 & 3401 & 425,5 & 11230,5 & 93,15 & 55,07 \\
\hline 19,9 & 19,5 & 22 & 3423 & 429 & 11659,5 & 93,76 & 57,18 \\
\hline 20,9 & 20,5 & 12 & 3435 & 246 & 11905,5 & 94,08 & 58,38 \\
\hline 21,9 & 21,5 & 10 & 3445 & 215 & 12120,5 & 94,36 & 59,44 \\
\hline 22,9 & 22,5 & 17 & 3462 & 382,5 & 12503 & 94,82 & 61,31 \\
\hline 23,9 & 23,5 & 11 & 3473 & 258,5 & 12761,5 & 95,12 & 62,58 \\
\hline 24,9 & 24,5 & 15 & 3488 & 367,5 & 13129 & 95,54 & 64,38 \\
\hline 25,9 & 25,5 & 11 & 3499 & 280,5 & 13409,5 & 95,84 & 65,76 \\
\hline 26,9 & 26,5 & 12 & 3511 & 318 & 13727,5 & 96,17 & 67,32 \\
\hline 27,9 & 27,5 & 13 & 3524 & 357,5 & 14085 & 96,52 & 69,07 \\
\hline 28,9 & 28,5 & 6 & 3530 & 171 & 14256 & 96,69 & 69,91 \\
\hline 29,9 & 29,5 & 6 & 3536 & 177 & 14433 & 96,85 & 70,78 \\
\hline 30,9 & 30,5 & 4 & 3540 & 122 & 14555 & 96,96 & 71,38 \\
\hline 31,9 & 31,5 & 7 & 3547 & 220,5 & 14775,5 & 97,15 & 72,46 \\
\hline 32,9 & 32,5 & 9 & 3556 & 292,5 & 15068 & 97,40 & 73,89 \\
\hline
\end{tabular}




\begin{tabular}{|c|c|c|c|c|c|c|c|}
\hline 33,9 & 33,5 & 5 & 3561 & 167,5 & 15235,5 & 97,53 & 74,71 \\
\hline 34,9 & 34,5 & 4 & 3565 & 138 & 15373,5 & 97,64 & 75,39 \\
\hline 35,9 & 35,5 & 6 & 3571 & 213 & 15586,5 & 97,81 & 76,44 \\
\hline 36,9 & 36,5 & 5 & 3576 & 182,5 & 15769 & 97,95 & 77,33 \\
\hline 37,9 & 37,5 & 4 & 3580 & 150 & 15919 & 98,06 & 78,07 \\
\hline 38,9 & 38,5 & 3 & 3583 & 115,5 & 16034,5 & 98,14 & 78,63 \\
\hline 39,9 & 39,5 & 5 & 3588 & 197,5 & 16232 & 98,27 & 79,60 \\
\hline 40,9 & 40,5 & 6 & 3594 & 243 & 16475 & 98,44 & 80,79 \\
\hline 42,9 & 42,5 & 6 & 3600 & 255 & 16730 & 98,60 & 82,04 \\
\hline 43,9 & 43,5 & 1 & 3601 & 43,5 & 16773,5 & 98,63 & 82,26 \\
\hline 44,9 & 44,5 & 3 & 3604 & 133,5 & 16907 & 98,71 & 82,91 \\
\hline 45,9 & 45,5 & 3 & 3607 & 136,5 & 17043,5 & 98,79 & 83,58 \\
\hline 46,9 & 46,5 & 3 & 3610 & 139,5 & 17183 & 98,88 & 84,27 \\
\hline 47,9 & 47,5 & 2 & 3612 & 95 & 17278 & 98,93 & 84,73 \\
\hline 49,9 & 49,5 & 1 & 3613 & 49,5 & 17327,5 & 98,96 & 84,97 \\
\hline 51,9 & 51,5 & 1 & 3614 & 51,5 & 17379 & 98,99 & 85,23 \\
\hline 52,9 & 52,5 & 3 & 3617 & 157,5 & 17536,5 & 99,07 & 86,00 \\
\hline 53,9 & 53,5 & 2 & 3619 & 107 & 17643,5 & 99,12 & 86,52 \\
\hline 54,9 & 54,5 & 4 & 3623 & 218 & 17861,5 & 99,23 & 87,59 \\
\hline 55,9 & 55,5 & 2 & 3625 & 111 & 17972,5 & 99,29 & 88,14 \\
\hline 56,9 & 56,5 & 1 & 3626 & 56,5 & 18029 & 99,32 & 88,41 \\
\hline 58,9 & 58,5 & 1 & 3627 & 58,5 & 18087,5 & 99,34 & 88,70 \\
\hline 59,9 & 59,5 & 3 & 3630 & 178,5 & 18266 & 99,42 & 89,58 \\
\hline 61,9 & 61,5 & 2 & 3632 & 123 & 18389 & 99,48 & 90,18 \\
\hline 63,9 & 63,5 & 1 & 3633 & 63,5 & 18452,5 & 99,51 & 90,49 \\
\hline 65,9 & 65,5 & 2 & 3635 & 131 & 18583,5 & 99,56 & 91,13 \\
\hline 67,9 & 67,5 & 1 & 3636 & 67,5 & 18651 & 99,59 & 91,46 \\
\hline 68,9 & 68,5 & 1 & 3637 & 68,5 & 18719,5 & 99,62 & 91,80 \\
\hline 75,9 & 75,5 & 2 & 3639 & 151 & 18870,5 & 99,67 & 92,54 \\
\hline 79,9 & 79,5 & 1 & 3640 & 79,5 & 18950 & 99,70 & 92,93 \\
\hline 85,9 & 85,5 & 1 & 3641 & 85,5 & 19035,5 & 99,73 & 93,35 \\
\hline 90,9 & 90,5 & 3 & 3644 & 271,5 & 19307 & 99,81 & 94,68 \\
\hline 99,9 & 99,5 & 1 & 3645 & 99,5 & 19406,5 & 99,84 & 95,17 \\
\hline 109,9 & 109,5 & 1 & 3646 & 109,5 & 19516 & 99,86 & 95,71 \\
\hline 119,9 & 119,5 & 1 & 3647 & 119,5 & 19635,5 & 99,89 & 96,29 \\
\hline 131,9 & 131,5 & 1 & 3648 & 131,5 & 19767 & 99,92 & 96,94 \\
\hline 133,9 & 133,5 & 1 & 3649 & 133,5 & 19900,5 & 99,95 & 97,59 \\
\hline
\end{tabular}




\begin{tabular}{|l|l|l|l|l|l|l|l|}
\hline 220,9 & 220,5 & 1 & 3650 & 220,5 & 20121 & 99,97 & 98,67 \\
\hline 270,9 & 270,5 & 1 & 3651 & 270,5 & 20391,5 & 100,00 & 100,00 \\
\hline Suma & & $\mathbf{3 6 5 1}$ & & $\mathbf{2 0 3 9 1 , 5}$ & & $\mathbf{6 7 1 5 , 3 4}$ & $\mathbf{4 9 6 1 , 6 9}$ \\
\hline
\end{tabular}

Fuente: Elaboración propia

Figura 1: Curvas de concentración, o de Lorenz, de Valencia y Salamanca, para el período 1951-2010. La mayor separación de la recta de equidistribución de Valencia, que posee un índice de Gini de 0,70, evidencia una mayor concentración diaria de la precipitación que en Salamanca, con índice de Gini 0,58

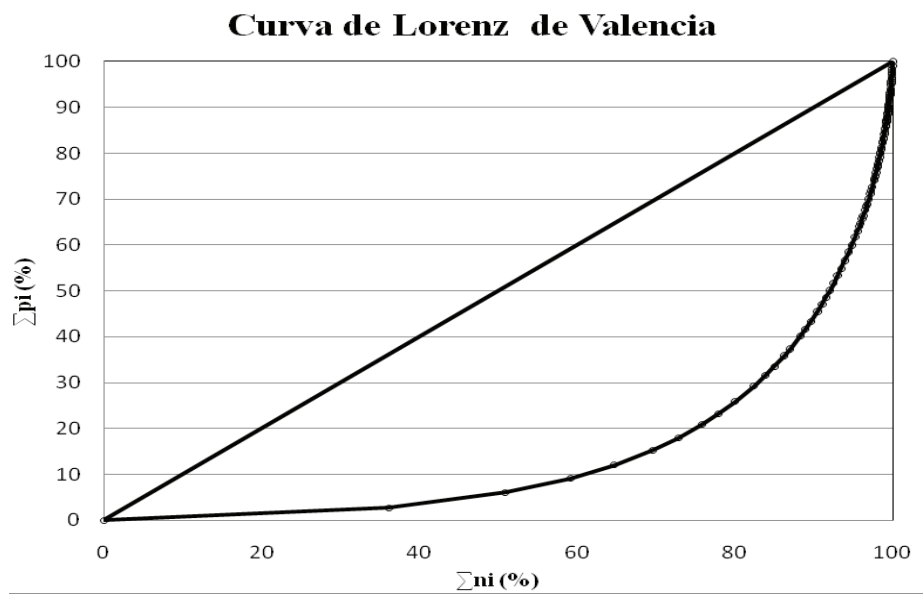

Curva de Lorenz de Salamanca

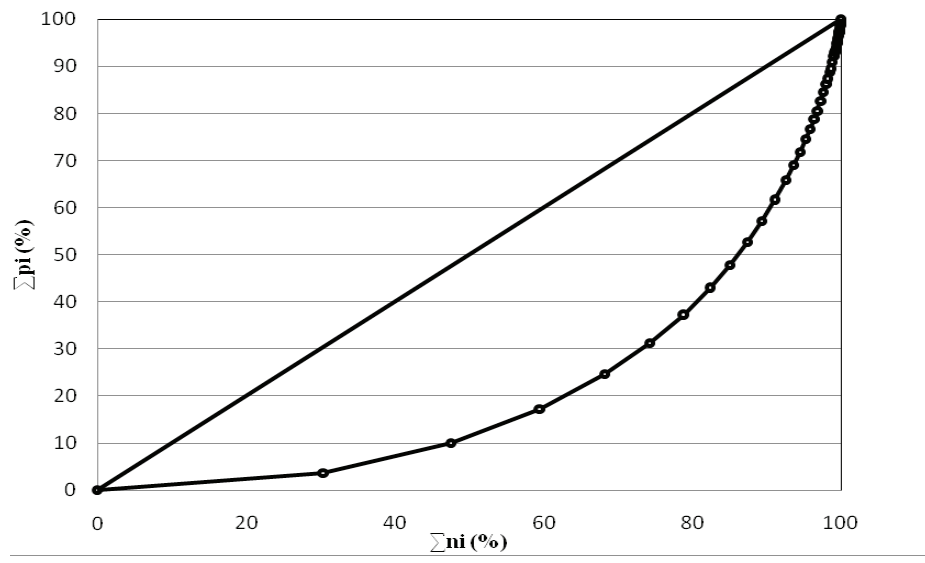

Fuente:Elaboración propia 
Sin embargo, puede perfeccionarse lo anterior, ajustando la distribución empírica de los porcentajes de precipitación aportados por los correspondientes porcentajes del número de días de lluvia mediante curvas exponenciales del tipo $\mathrm{y}=\mathrm{axe}^{\mathrm{bx}}$ (Guilló y Puigcerver, 1970). Entonces puede definirse un índice similar al de Gini sobre la curva exponencial. El índice CI se define como el cociente entre el área (S) delimitada por la recta de equidistribución, la curva exponencial de ajuste y $x=100$, y el área del triángulo definido por la recta de equidistribución, el eje de abscisas y $x=100$,

$$
\mathrm{CI}=\mathrm{S} / 5000
$$

Con valores extremos ideales de 0 (todas las cantidades diarias iguales) y 1 (un solo día de precipitación). El índice CI representa el tanto por uno del área comprendida entre la recta de equidistribución, la curva exponencial y la ordenada $X=100$, y el área del triángulo mencionado. Nótese que cuanto mayor sea el valor de CI más peso tienen unos pocos días muy lluviosos en el total pluviométrico.

Las constantes $a$ y $b$ de la ecuación (1) se determinan mediante mínimos cuadrados, de la siguiente forma:

$$
\begin{aligned}
\text { h } a & =\frac{\sum x_{1}^{2} \sum \mathrm{h} y_{1}+\sum x_{1} \sum x_{1} \mathrm{~h} x_{1}-\sum x_{1}^{2} \sum \mathrm{h} x_{1}-\sum x_{1} \sum x_{1} \mathrm{~h} y_{1}}{N \sum x_{1}^{2}-\left(\sum x_{1}\right)^{2}} \\
b & =\frac{N \sum x_{1} \sum \mathrm{h} y_{1}+\sum x_{1} \sum \mathrm{h} x_{1}-N \sum x_{1} \mathrm{~h} x_{1}-\sum x_{1} \sum \mathrm{h} y_{1}}{N \sum x_{1}^{2}-\left(\sum x_{1}\right)^{2}}
\end{aligned}
$$

El uso de clases de $1 \mathrm{~mm}$ de longitud para el cálculo de CI puede considerarse costoso, no por el tiempo de cálculo, hoy mínimo, sino por la demanda de datos diarios de alta resolución y calidad. En sí, el CI es muy sensible a la calidad de los datos. Cuando en un observatorio no se ha considerado o no se han registrado las cantidades más pequeñas, de unas décimas de mm (excluidos los valores inapreciables), o se han juntado las cantidades de dos días consecutivos, el valor de CI obtenido resulta anómalo, en general inferior al que correspondería si se hubieran contabilizado todos los datos diarios. En este caso, hay que prescindir del observatorio.

Para tratar de "salvar" observatorios, puede abordarse el análisis de la concentración diaria con clases de $5 \mathrm{~mm}$ o de $10 \mathrm{~mm}$. Con el mismo procedimiento de cálculo indicado anteriormente pueden hallarse índices de concentración diaria en base a curvas exponenciales del mismo tipo y clases de $5 \mathrm{~mm}$, que se denominará CI5, y de $10 \mathrm{~mm}$, CI10. También podrán calcularse los índices de Gini, sobre los valores empíricos (X, Y) de clases de $5 \mathrm{~mm}$, que se denominará IG5, y de $10 \mathrm{~mm}$, IG10. Los valores de IG5, IG10 e IG, en adelante, IG1, verificarán:

$$
\text { IG1 > IG5 > IG10, }
$$

Como puede deducirse de la figura 2. Igualmente, ha de cumplirse en general:

$$
\mathrm{CI} 1>\mathrm{CI} 5>\mathrm{CI} 10
$$


Como hipótesis de trabajo, se asume en primer lugar que las correlaciones entre todos los índices, empíricos (los de Gini) y ajustados (los de concentración usando la curva exponencial), indicados han de ser elevadas, dadas sus definiciones. En segundo lugar, se plantea el aumento de la concentración diaria de la precipitación, y, en consecuencia, de los índices definidos entre el primer y el segundo subperíodo, acorde con algunas de las tendencias climáticas observadas en la cuenca mediterránea en las últimas décadas.

Figura 2: Curvas de concentración, o de Lorenz, de Alicante usando clases de 1, 5 y $10 \mathrm{~mm}$, para el período 1951-2010

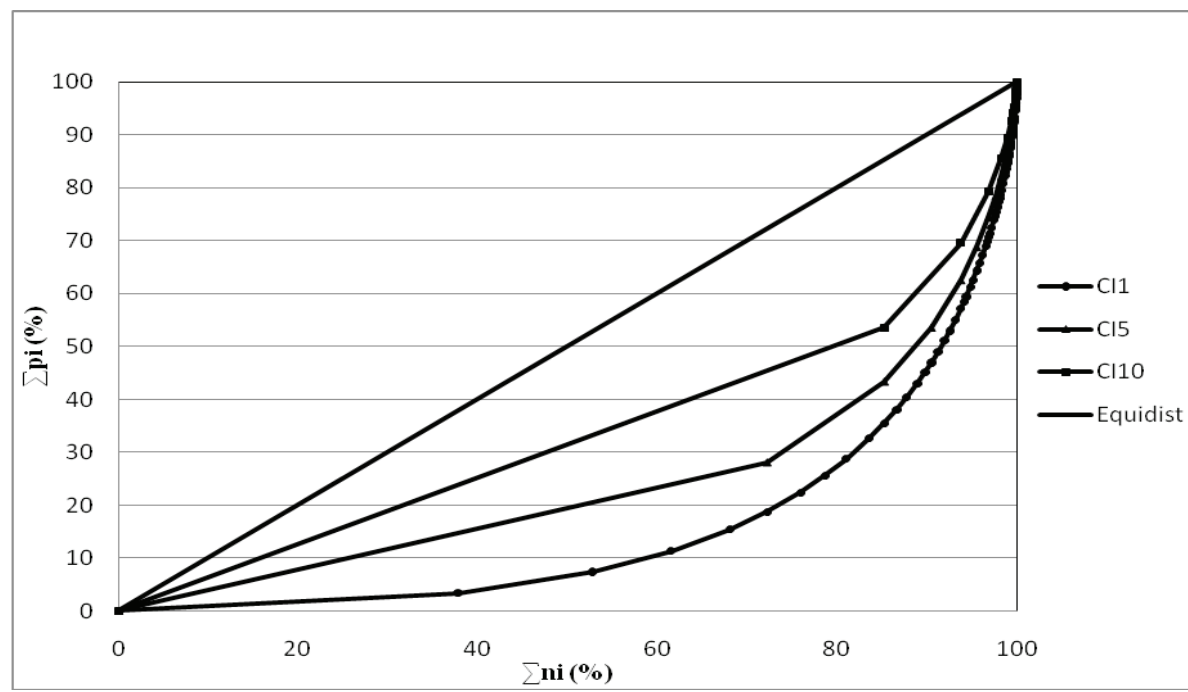

Fuente: Elaboración propia

\section{RESULTADOS Y DISCUSIÓN: COMPARACIÓN DE LA CONCENTRACIÓN DIARIA SEGÚN LOS DIFERENTES ÍNDICES}

Se han hallado los valores de los índices CI1, CI5, CI10, IG1, IG5 e IG10 de los 32 observatorios para el período 1951-2010 (cuadro 2) y los subperíodos 1951-1980 y 1981-2010. Véase que para el período completo CI1 >CI5>CI10 en todos los casos e, igualmente, IG1>IG5>IG10. En los dos subperíodos también se verifican las desigualdades anteriores.

Si se comparan CI1 e IG1, los valores, con dos decimales, son iguales o el índice de Gini supera al primero en una centésima, con la única excepción de Vigo, en que ocurre al revés. En los dos subperíodos también los valores de CI1 e IG1 son iguales o el índice de Gini supera al primero en una centésima, salvo en Córdoba, en 19511980 , en que ocurre al revés. 
El rango de valores aumenta a medida que aumenta la longitud de las clases. Así, para el período completo, los extremos del CI1 se sitúan en 0,57 y 0,69 (12 centésimas de variación), del CI5, en 0,51 y 0,65 (14 centésimas) y del CI10, en 0,46 y 0,60 (14 centésimas). En los casos del IG1 los extremos son 0,57 y 0,70 (13 centésimas), del CI5, 0,38 y 0,56 (18 centésimas) y del IG10, 0,19 y 0,47 ( 28 centésimas).

Cuadro 2: Valores de los índices CI1, CI5, CI10, IG1, IG5 e IG10 de los 32 observatorios analizados para el período 1951-2010

\begin{tabular}{|c|c|c|c|c|c|c|}
\hline Observatorios & CI1 & CI5 & CI10 & IG1 & IG5 & IG10 \\
\hline Albacete & 0,60 & 0,54 & 0,50 & 0,61 & 0,45 & 0,26 \\
\hline Alicante & 0,68 & 0,62 & 0,58 & 0,69 & 0,52 & 0,34 \\
\hline Almería & 0,63 & 0,57 & 0,51 & 0,64 & 0,47 & 0,28 \\
\hline Ávila & 0,59 & 0,52 & 0,48 & 0,60 & 0,38 & 0,19 \\
\hline Barcelona & 0,65 & 0,60 & 0,55 & 0,66 & 0,56 & 0,42 \\
\hline Burgos & 0,59 & 0,52 & 0,47 & 0,59 & 0,42 & 0,23 \\
\hline Cáceres & 0,58 & 0,53 & 0,48 & 0,58 & 0,48 & 0,31 \\
\hline Ciudad Real & 0,57 & 0,51 & 0,48 & 0,57 & 0,45 & 0,27 \\
\hline Córdoba & 0,59 & 0,54 & 0,51 & 0,59 & 0,52 & 0,40 \\
\hline Cuenca & 0,57 & 0,51 & 0,46 & 0,57 & 0,45 & 0,27 \\
\hline Gerona & 0,63 & 0,59 & 0,55 & 0,64 & 0,56 & 0,44 \\
\hline Gijón & 0,60 & 0,54 & 0,50 & 0,60 & 0,48 & 0,32 \\
\hline Granada & 0,57 & 0,51 & 0,47 & 0,57 & 0,43 & 0,25 \\
\hline Huelva & 0,60 & 0,55 & 0,51 & 0,60 & 0,52 & 0,38 \\
\hline Huesca & 0,60 & 0,54 & 0,50 & 0,61 & 0,49 & 0,33 \\
\hline La Coruña & 0,57 & 0,51 & 0,47 & 0,57 & 0,46 & 0,30 \\
\hline León & 0,57 & 0,51 & 0,48 & 0,58 & 0,44 & 0,27 \\
\hline Logroño & 0,60 & 0,54 & 0,49 & 0,61 & 0,40 & 0,20 \\
\hline Madrid & 0,60 & 0,52 & 0,47 & 0,60 & 0,44 & 0,26 \\
\hline Murcia & 0,67 & 0,61 & 0,56 & 0,68 & 0,50 & 0,32 \\
\hline Orense & 0,57 & 0,52 & 0,47 & 0,58 & 0,48 & 0,33 \\
\hline Pamplona & 0,59 & 0,54 & 0,50 & 0,60 & 0,47 & 0,30 \\
\hline Salamanca & 0,57 & 0,51 & 0,47 & 0,58 & 0,39 & 0,20 \\
\hline San Fernando & 0,60 & 0,55 & 0,51 & 0,61 & 0,52 & 0,39 \\
\hline San Sebastián & 0,60 & 0,54 & 0,50 & 0,60 & 0,52 & 0,40 \\
\hline Sevilla & 0,59 & 0,55 & 0,50 & 0,59 & 0,52 & 0,40 \\
\hline Soria & 0,57 & 0,51 & 0,46 & 0,58 & 0,42 & 0,23 \\
\hline Tortosa & 0,69 & 0,64 & 0,59 & 0,70 & 0,56 & 0,40 \\
\hline
\end{tabular}




\begin{tabular}{|l|l|l|l|l|l|l|}
\hline Valencia & 0,69 & 0,65 & 0,60 & 0,70 & 0,56 & 0,40 \\
\hline Valladolid & 0,59 & 0,52 & 0,47 & 0,59 & 0,41 & 0,21 \\
\hline Vigo & 0,59 & 0,54 & 0,51 & 0,58 & 0,54 & 0,47 \\
\hline Zaragoza & 0,62 & 0,56 & 0,52 & 0,63 & 0,43 & 0,23 \\
\hline
\end{tabular}

Fuente: Elaboración propia

En el cuadro 3 se muestran los valores del coeficiente de correlación de Pearson entre los seis índices de los 32 observatorios analizados para el período completo (19512010) y para los dos subperíodos de 30 años que lo componen. Los valores muestran correlaciones muy altas, en todos los casos significativas $(\mathrm{p}<0,000)$, entre CI1, CI5, CI10 e IG1, y claramente inferiores en las que participan IG5 e IG10 con los demás.

Cuadro 3: Valores del coeficiente de correlación de Pearson entre los diferentes índices de concentración diaria de 32 observatorios de la España peninsular durante el período 19512010 y los subperíodos 1951-1980 y 1981-2010. (Todos los valores son positivos y significativos, $\mathrm{p}<0,000)$

\begin{tabular}{|c|c|c|c|c|c|c|}
\hline 1951-2010 & CI1 & CI5 & CI10 & IG1 & IG5 & IG10 \\
\hline CI1 & 1 & 0,98 & 0,95 & 0,998 & 0,59 & 0,40 \\
\hline CI5 & & 1 & 0,99 & 0,97 & 0,71 & 0,54 \\
\hline CI10 & & & 1 & 0,94 & 0,76 & 0,61 \\
\hline IG1 & & & & 1 & 0,56 & 0,36 \\
\hline IG5 & & & & & 1 & 0,97 \\
\hline IG10 & & & & & & 1 \\
\hline 1951-1980 & CI1 & CI5 & CI10 & IG1 & IG5 & IG10 \\
\hline CI1 & 1 & 0,96 & 0,9 & 0,998 & 0,56 & 0,34 \\
\hline CI5 & & 1 & 0,97 & 0,96 & 0,70 & 0,51 \\
\hline CI10 & & & 1 & 0,89 & 0,78 & 0,62 \\
\hline IG1 & & & & 1 & 0,52 & 0,30 \\
\hline IG5 & & & & & 1 & 0,96 \\
\hline IG10 & & & & & & 1 \\
\hline 1981-2010 & CI1 & CI5 & CI10 & IG1 & IG5 & IG10 \\
\hline CI1 & 1 & 0,96 & 0,92 & 0,996 & 0,58 & 0,43 \\
\hline CI5 & & 1 & 0,98 & 0,96 & 0,71 & 0,57 \\
\hline CI10 & & & 1 & 0,92 & 0,75 & 0,62 \\
\hline IG1 & & & & 1 & 0,57 & 0,40 \\
\hline IG5 & & & & & 1 & 0,98 \\
\hline IG10 & & & & & & 1 \\
\hline
\end{tabular}

Fuente: Elaboración propia 
En particular CI1 e IG1 presentan correlaciones prácticamente perfectas. En consecuencia, los mapas resultantes de representar los valores de cada uno de los índices de los 32 observatorios no muestran grandes diferencias, con el sur de Cataluña y la Comunidad Valenciana como áreas con los valores más elevados, excepto los de IG5 e IG10, en que se difumina este hecho (ver las figuras 3, 4 y 5).

Figura 3: Isopletas de los índices CI1, CI5, CI10, IG1, IG5 e IG10 en la España peninsular, para el período 1951-2010

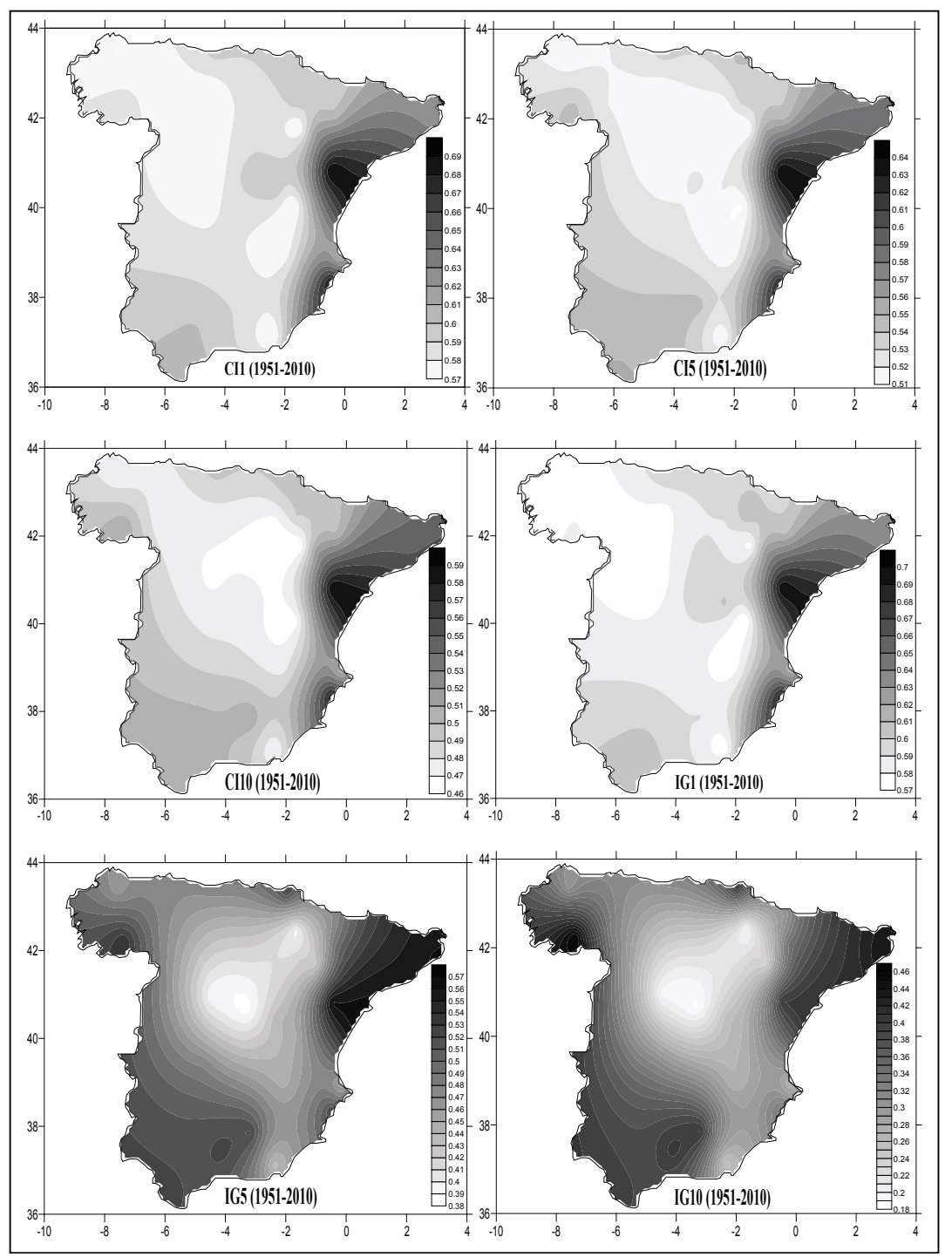

Fuente: Elaboración propia 
Figura 4: Isopletas de los índices CI1, CI5, CI10, IG1, IG5 e IG10 en la España peninsular, para el período 1951-1980

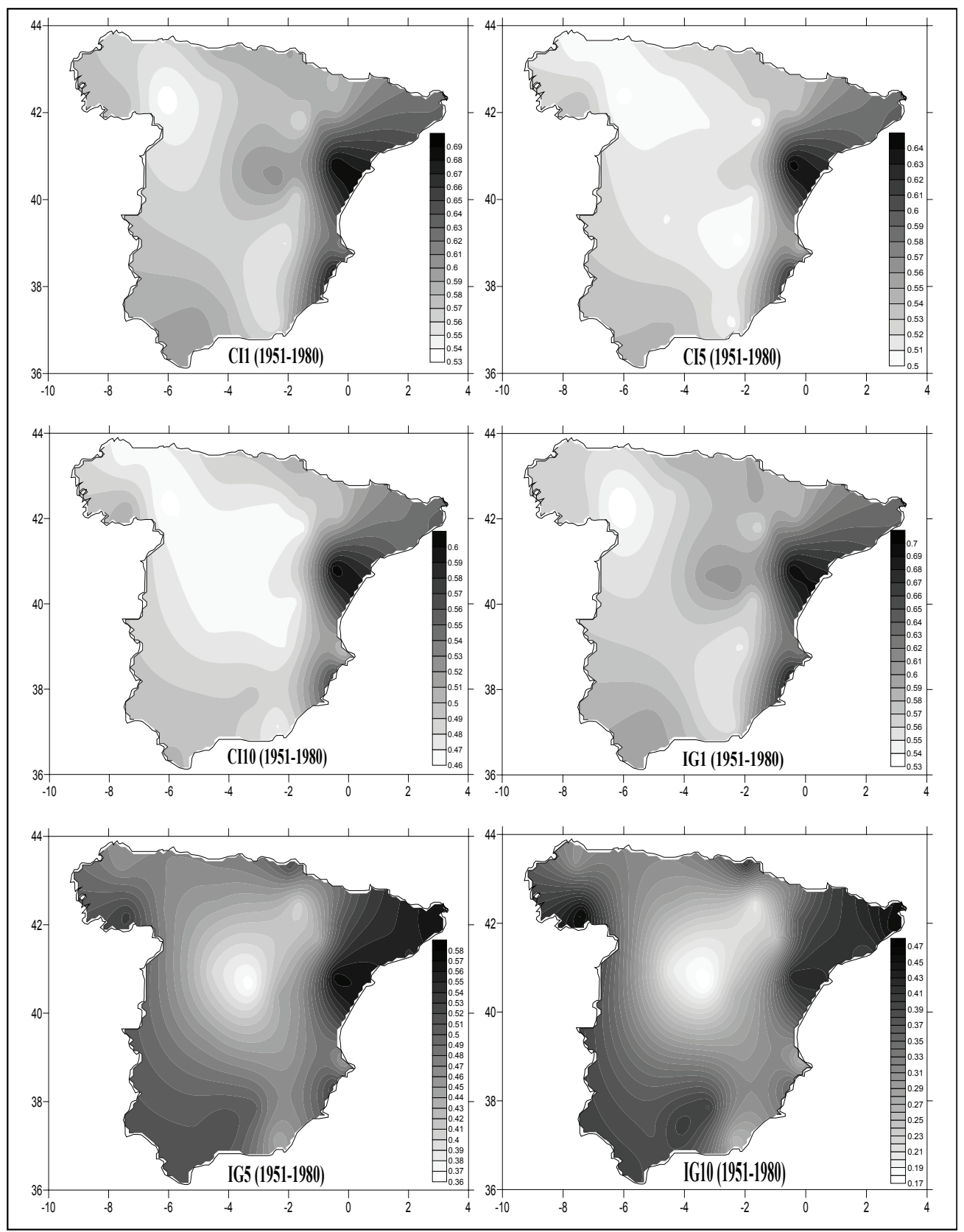

Fuente: Elaboración propia 
Figura 5: Isopletas de los índices CI1, CI5, CI10, IG1, IG5 e IG10 en la España peninsular, para el período 1981-2010

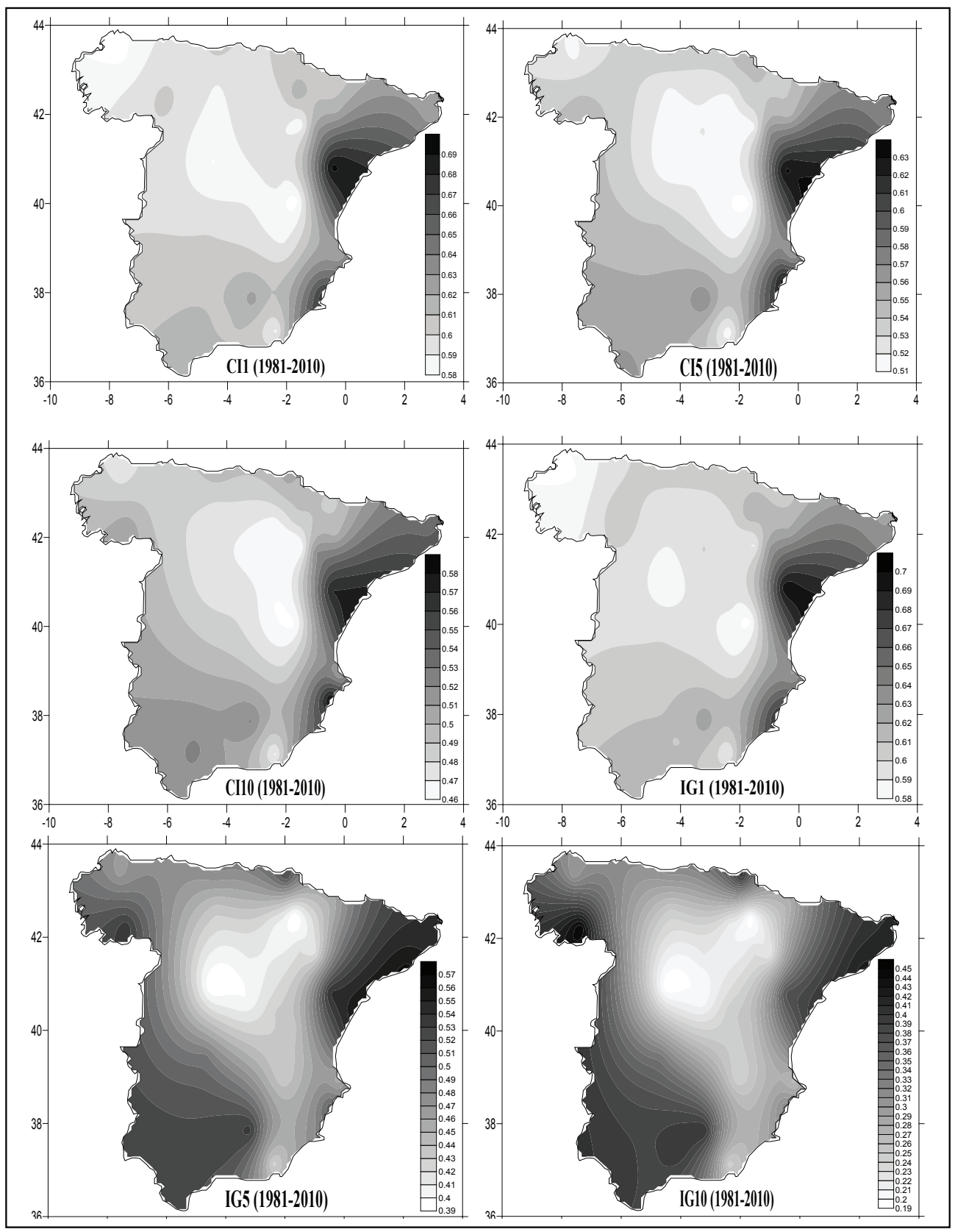

Fuente: Elaboración propia 
Los mapas de CI1, CI5, CI10 e IG1 son muy parecidos a los hallados en (MartínVide, 2004) y (Sánchez Lorenzo y Martín-Vide, 2006). Los valores más elevados de CI1, para el período completo, son los de Valencia y Tortosa $(0,69)$, seguidos por Alicante $(0,68)$ y Murcia $(0,67)$, por lo que en la parte central de la franja oriental ibérica se localizan las concentraciones diarias más altas, ya intuidas en el trabajo pionero de (Martín-Vide, 1987).

A la vista del cuadro 3 se concluye que la primera hipótesis se verifica con claridad. Sin embargo, sólo 4 de los seis índices, los CI1, CI5, CI10 e IG1 reflejan nítidamente las diferencias espaciales en la concentración diaria de la precipitación sobre el territorio estudiado. Si se dispone sólo de valores pluviométricos diarios clasificados en clases de $5 \mathrm{o}$, incluso de $10 \mathrm{~mm}$, y se sabe que éstos son de calidad y que están completos, no hay ninguna objeción para evaluar la concentración diaria de la precipitación con los índices CI5 o CI10, aunque naturalmente el rango de valores es diferente. En cambio, los índices IG5 e IG10, en especial el último, encumbran áreas del oeste peninsular, en especial del suroeste de Galicia (Vigo) a los primeros lugares en el ránking de concentración diaria de la precipitación de España. Así, por ejemplo, para el período 1951-2010, Vigo, con 0,47, tiene el valor más elevado de IG10, hecho que se debe a que las clases de $10 \mathrm{~mm}$ son excesivamente groseras para discriminar adecuadamente la concentración diaria en lugares muy lluviosos, como es el caso de la ciudad gallega.

Los valores de CI1 hallados para los observatorios españoles, en comparación con los obtenidos con el mismo método para Irán de (Alijani et al, 2008) y China de (Zang et al, 2009) permiten afirmar que la concentración diaria de la precipitación en la franja oriental ibérica es elevada. Los nuevos análisis venideros sobre otros países y ámbitos climáticos permitirán establecer comparaciones absolutas más precisas.

Finalmente, desde un punto de vista metodológico, podrán admitirse como buenos los valores de los índices de concentración CI5 y CI10 de observatorios sin una gran precisión en el registro de las décimas de precipitación, aunque con los registros completos o cuasi completos, dado que habrá pocas asignaciones erróneas de valores entre clases, al ser éstas relativamente escasas.

\section{RESULTADOS Y DISCUSIÓN: VARIACIÓN DE LA CONCENTRACIÓN DIARIA ENTRE LOS PERÍODOS 1951-1980 Y 1981-2010}

Se han comparado los valores de los diferentes índices de los 32 observatorios analizados de los subperíodos 1951-1980 y 1981-2010. Los resultados se resumen en el cuadro 4 . 
Cuadro 4: Diferencia entre los valores de los índices CI1, CI5, CI10, IG1, IG5 e IG10 del subperíodo 1981-2010 menos los del subperíodo 1951-1980 de los 32 observatorios analizados

\begin{tabular}{|l|c|c|c|c|c|c|}
\hline $\begin{array}{l}\text { Diferencia valores (1981-2010 } \\
\text { menos 1951-1980) }\end{array}$ & CI1 & CI5 & CI10 & IG1 & IG5 & IG10 \\
\hline Aumento & 26 & 19 & 18 & 26 & 12 & 7 \\
\hline Igualdad & 2 & 10 & 6 & 2 & 5 & 3 \\
\hline Disminución & 4 & 3 & 8 & 4 & 15 & 22 \\
\hline & & & & & & \\
\hline
\end{tabular}

Fuente: Elaboración propia

Los índices CI1 e IG1 muestran un claro predominio de los aumentos de la concentración al pasar del primer al segundo subperíodo: en más del 80\% de los observatorios se da un incremento. Sólo en el este, Alicante y Valencia, y el centro, Madrid y Ávila, con el CI1 (Alicante y Girona, y Madrid y Ávila, con el IG1) han visto disminuir el valor del índice. Los aumentos en algunos observatorios cabe considerarlos sustantivos, entre 4 y 7 centésimas para el CI1 (hasta 8 para el IG1), casi siempre en la mitad occidental del territorio, como son los casos de Orense, Córdoba, Cáceres y Granada.

Los índices CI5 y CI10 confirman el predominio de aumentos de la concentración diaria, aunque en un porcentaje de observatorios inferior, de algo menos del $60 \%$. En cambio, los índices IG5 e IG10 muestran un predominio de descensos, en especial el IG10, con disminuciones de valor en más de dos terceras partes de los observatorios. Este comportamiento discrepante de IG5 e IG10, probablemente relacionado con su incapacidad para discriminar con precisión la concentración diaria, según se ha visto en el apartado anterior, los excluye para el análisis de tendencias.

En consecuencia, se confirma también la segunda hipótesis, relativa a un incremento de la concentración diaria entre el primer y el segundo subperíodo, en consonancia con algunas de las tendencias halladas en el comportamiento extremo de la precipitación en la cuenca del Mediterráneo (IPCC, 2007; Gallego et al., 2006), aunque tales análisis y, sobre todo, sus proyecciones futuras presentan una acusada incertidumbre.

\section{CONCLUSIONES}

La concentración diaria de la precipitación en la franja oriental de la España peninsular es, a la luz de los estudios disponibles, elevada, con valores del índice de concentración (Concentration Index, CI) próximos a 0,70, lo que refleja el hecho de que unos pocos días muy lluviosos aportan un elevado porcentaje de la precipitación anual. 
Si en lugar de usar clases de $1 \mathrm{~mm}$ de longitud para el cálculo del índice de concentración (CI1) o del correspondiente índice de Gini (IG1), se emplean clases de 5 $\mathrm{mm}$ (CI5 e IG5) y de $10 \mathrm{~mm}$ (CI10 e IG10), los mapas de CI5 y CI10 son muy parecidos a los realizados con clases de $1 \mathrm{~mm}$. Aunque el rango de valores es diferente, y se cumplen, además, las desigualdades CI1 $>$ CI5 $>$ CI10 e IG1 $>$ IG5 $>$ IG10, 4 de los seis índices, CI1, CI5, CI10 e IG1, están alta y significativamente correlacionados, con coeficientes de correlación de Pearson en general superiores a $+0,90$, e incluso cercanos a 1, en el caso de CI1 e IG1. En consecuencia, no hay objeción para emplear clases de 5 y $10 \mathrm{~mm}$ y los índices CI5 y CI10, pero no los IG5 e IG10, en el análisis de la concentración diaria, que puede permitir el estudio de algún observatorio sin precisión en el registro de las décimas de precipitación. El uso, por tanto, de las curvas exponenciales y los índices CI5 y CI10 derivados es superior a las curvas empíricas de Lorenz.

Al pasar del subperíodo 1951-1980 al 1981-2010 la concentración diaria de la precipitación, según la mayoría de los índices usados, ha aumentado en un número sustancial de observatorios.

\section{AGRADECIMIENTOS}

Este trabajo ha sido posible gracias a la financiación por el Ministerio de Ciencia e Innovación como forma parte del proyecto CGL2008-06129-C02-01/CLI, 2009-2011 y la beca FPI de que disfruta el primer autor. Los datos han sido proporcionados por la Agencia Estatal de Meteorología de España (AEMET).

\section{BIBLIOGRAFÍA}

ALIJANI, B. J., O'BRIEN y YARNAL, B. (2008): Spatial analysis of precipitation intensity and concentration in Iran. Theor. Appl. Climatol 94: 107-124 pp.

BEGUERÍA, S.; VICENTE-SERRANO, M.; LÓPEZ-MORENO, J.I. y GARCÍARUIZ, J.M. (2009): Annual and seasonal mapping of peak intensity, magnitude and duration of extreme precipitation events across a climatic gradient, northeast Spain. Internation Journal of Climatology, 29, 1759-1779.

BROOKS, C.E.P. y CARRUTHERS, N. (1953): Handbooks of statistical methods in Meteorology. Londres, Meteorological Office.

ELÍAS CASTILLO, F. y RUIZ BERTRÁN (1979): «precipitaciones máximas en España», servicio de publicaciones agrarias, Ministerio de Agricultura, $545 \mathrm{pp}$.

GALLEGO, M. C.; GARCÍA, J. A.; VAQUERO, J. M. y MATEOS, V. L. (2006): Changes in frequency and intensity of daily precipitation over the Iberian Peninsula, Journal of Geophysical Research, 111, D24105, doi: 10.1029/2006JD007280.

GIL OLCINA, A. y OLCINA, J (1997): Climatología General, Barcelona, Ariel. 
GUILLÓ, A.M. y PUIGCERVER, M. (1970): «Sobre las contribuciones relativas de las precipitaciones local y generalizada a la precipitación total en Cataluña», en Revista de Geofísica, XXIX, 3, pp. 205-216.

IPCC (2007): Cuarto Informe IPCC, www.ipcc.ch.

MARTÍN-VIDE, J. (1987): Característiques climatològiques de la precipitació en la franja costera mediterrània de la Península Ibèrica, Barcelona, Institut Cartogràfic de Catalunya.

MARTÍN-VIDE, J. (2004): Spatial distribution of daily precipitation concentration index in Peninsular Spain. International Journal of Climatology, 24, 959-971.

MARTÍN-VIDE, J. (2011): Estructura temporal fina y patrones espaciales de la precipitación en la España peninsular. Memorias de la Real Academia de Ciencias y Artes de Barcelona, LXV, 3, 119-162.

MARTÍN-VIDE, J. y OLCINA, J. (2001): Climas y tiempos de España, Madrid, Alianza Editorial.

OLCINA, J. (2006): ¿Riesgos Naturales? I. Sequías e inundaciones. Barcelona, Davinci continental.

PITA LÓPEZ, M $M^{a}$ F. (2001): “Sequías en la Cuenca del Guadalquivir”, pp. 303-343, en GIL OLCINA, A. y MORALES GIL (edit.): Causas y consecuencias de las sequías en España, Caja de Ahorros del Mediterráneo e Instituto Universitario de Geografía (Universidad de Alicante), 574 pp.

SÁNCHEZ-LORENZO y MARTÍN-VIDE, J. (2006): Distribución espacial de la concentración pluviométrica diaria en la Península Ibérica. $5^{\text {a }}$ Asamblea Hispanoportuguesa de Geodesia y Geofísica, Sevilla.

ZHANG, Q., XU C-Y., GEMMER M., CHEN YQ., LIU C-L. (2009): Changing properties of precipitation concentration in the Pearl River basin, China. Stoch Environ Res Risk Assess (2009) 23:377-385. 